\title{
On the injectivity of Wachspress and mean value mappings between convex polygons
}

\author{
Michael S. Floater and Jiří Kosinka
}

\begin{abstract}
Wachspress and mean value coordinates are two generalizations of triangular barycentric coordinates to convex polygons and have recently been used to construct mappings between polygons, with application to curve deformation and image warping. We show that Wachspress mappings between convex polygons are always injective but that mean value mappings can fail to be so in extreme cases.

Keywords: Barycentric coordinates, Wachspress coordinates, mean value coordinates, injectivity.

Math Subject Classification: 65D17, 26B10
\end{abstract}

\section{Introduction}

There has recently been considerable interest in using generalized barycentric coordinates to deform shapes, when modelling and processing geometry: shapes defined either by curves or surfaces $[22,14,18]$. Most of these methods focus on two kinds of barycentric coordinates: Wachspress coordinates $[20,21,13]$ and mean value coordinates $[7,9,14,10,5]$. Further work on generalized barycentric coordinates can be found in $[2,8,11,15,16,17,19]$. In the planar case, such barycentric coordinates can be used to map one polygon into another. So far, however, nothing seems to be known about when such mappings are injective, even though this is clearly an important property when these mappings are used in practice.

In this paper we investigate the injectivity of Wachspress and mean value mappings between planar convex polygons. We show that Wachspress mappings are always injective. However, we have found examples of mean value mappings that are not injective when the polygons have five or more vertices. 

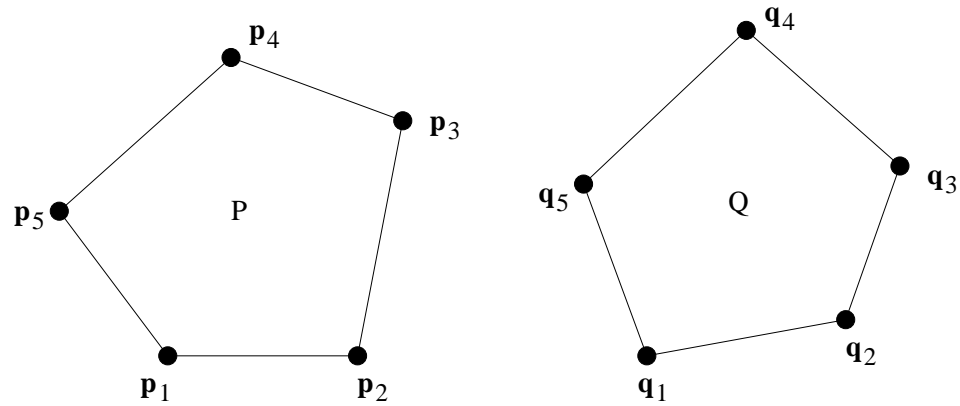

Figure 1: Two convex polygons.

\section{Barycentric mappings}

Let $P, Q \subset \mathbb{R}^{2}$ be strictly convex polygons with vertices $\mathbf{p}_{1}, \mathbf{p}_{2}, \ldots, \mathbf{p}_{n}$ and $\mathbf{q}_{1}, \mathbf{q}_{2}, \ldots, \mathbf{q}_{n}$ respectively, ordered anticlockwise, with $n \geq 3$. By strictly, we mean that no three vertices in the polygon are collinear. Figure 1 shows an example with $n=5$. We view both polygons as open sets of $\mathbb{R}^{2}$ and denote their boundaries by $\partial P$ and $\partial Q$ and their closures by $\bar{P}$ and $\bar{Q}$. We would like to define a smooth mapping $\mathbf{f}: \bar{P} \rightarrow \bar{Q}$. One way is to use barycentric coordinates. Let $\lambda_{1}, \lambda_{1}, \ldots, \lambda_{n}: \bar{P} \rightarrow \mathbb{R}$ be a sequence of nonnegative functions such that

$$
\begin{gathered}
\sum_{i=1}^{n} \lambda_{i}(\mathbf{x})=1, \quad \mathbf{x} \in \bar{P}, \\
\sum_{i=1}^{n} \lambda_{i}(\mathbf{x}) \mathbf{p}_{i}=\mathbf{x}, \quad \mathbf{x} \in \bar{P} .
\end{gathered}
$$

We call $\lambda_{1}, \ldots, \lambda_{n}$ a set of barycentric coordinates for $P$. If $n=3, P$ is a triangle, and the equations (1-2) have, by Cramer's rule, the unique solution

$$
\lambda_{1}(\mathbf{x})=\frac{A\left(\mathbf{x}, \mathbf{p}_{2}, \mathbf{p}_{3}\right)}{A\left(\mathbf{p}_{1}, \mathbf{p}_{2}, \mathbf{p}_{3}\right)}, \quad \lambda_{2}(\mathbf{x})=\frac{A\left(\mathbf{p}_{1}, \mathbf{x}, \mathbf{p}_{3}\right)}{A\left(\mathbf{p}_{1}, \mathbf{p}_{2}, \mathbf{p}_{3}\right)}, \quad \lambda_{3}(\mathbf{x})=\frac{A\left(\mathbf{p}_{1}, \mathbf{p}_{2}, \mathbf{x}\right)}{A\left(\mathbf{p}_{1}, \mathbf{p}_{2}, \mathbf{p}_{3}\right)} .
$$

where $A(\mathbf{p}, \mathbf{q}, \mathbf{r})$ denotes the signed area of the triangle $[\mathbf{p}, \mathbf{q}, \mathbf{r}]$,

$$
A(\mathbf{p}, \mathbf{q}, \mathbf{r})=\frac{1}{2}\left|\begin{array}{ccc}
1 & 1 & 1 \\
p^{1} & q^{1} & r^{1} \\
p^{2} & q^{2} & r^{2}
\end{array}\right| .
$$


For $n \geq 4$, there are several possible choices of barycentric coordinates. One is the construction that goes back to Wachspress. For $\mathrm{x} \in \bar{P}$ let

$$
\lambda_{i}(\mathbf{x})=w_{i}(\mathbf{x}) / \sum_{j=1}^{n} w_{j}(\mathbf{x}),
$$

with

$$
w_{i}(\mathbf{x})=C_{i} \prod_{j \neq i-1, i} A_{j}(\mathbf{x}) .
$$

where $C_{i}=A\left(\mathbf{p}_{i-1}, \mathbf{p}_{i}, \mathbf{p}_{i+1}\right)$, and $A_{j}(\mathbf{x})=A\left(\mathbf{x}, \mathbf{p}_{j}, \mathbf{p}_{j+1}\right)$; see $[20,21,13]$. Here and throughout the paper we view the index $i$ in $\mathbf{p}_{i}, \mathbf{q}_{i}, A_{i}, w_{i}$ and so on cyclically with respect to $n$.

If $\mathbf{x}$ is not on the boundary of $P$ we can replace (5) by

$$
w_{i}(\mathbf{x})=\frac{C_{i}}{A_{i-1}(\mathbf{x}) A_{i}(\mathbf{x})}
$$

without altering $\lambda_{i}$. We will use this local expression later. A simple proof that the $\lambda_{i}$ satisfy (2) is given in the appendix of [13]. Another choice is the mean value coordinates which, for $\mathbf{x} \in P$, are given by (4) with

$$
w_{i}(\mathbf{x})=\frac{1}{\left\|\mathbf{x}-\mathbf{p}_{i}\right\|}\left(\tan \left(\alpha_{i-1}(\mathbf{x}) / 2\right)+\tan \left(\alpha_{i}(\mathbf{x}) / 2\right)\right),
$$

where $\|\cdot\|$ is the Euclidean norm in $\mathbb{R}^{2}$ and $\alpha_{i}(\mathbf{x})$ denotes the angle at $\mathbf{x}$ in the triangle $\left[\mathbf{x}, \mathbf{p}_{i}, \mathbf{p}_{i+1}\right]$; see [7]. These mean value coordinates $\lambda_{i}$ extend continuously to the boundary $\partial P$ in the same way as Wachspress and all other barycentric coordinates. Using (1-2), it can be shown (see [8]) that if

$$
\mathbf{x}=(1-\mu) \mathbf{p}_{\ell}+\mu \mathbf{p}_{\ell+1},
$$

for some $\ell$ and some $\mu \in[0,1]$, with indexes treated cyclically, then

$$
\lambda_{\ell}(\mathbf{x})=1-\mu, \quad \lambda_{\ell+1}(\mathbf{x})=\mu, \quad \text { and } \quad \lambda_{i}(\mathbf{x})=0 \quad \text { for } i \neq \ell, \ell+1 .
$$

Consider next a barycentric mapping from $P$ to $Q$. We define the mapping f : $\bar{P} \rightarrow \mathbb{R}^{2}$ by

$$
\mathbf{f}(\mathbf{x})=\sum_{i=1}^{n} \lambda_{i}(\mathbf{x}) \mathbf{q}_{i}, \quad \mathbf{x} \in \bar{P}
$$


Then (1) implies that $\mathbf{f}(\mathbf{x})$ is a convex combination of the points $\mathbf{q}_{i}$ and so $\mathbf{f}(\bar{P}) \subset \bar{Q}$. Further, by (9), if $\mathbf{x}$ is the boundary point (8) then

$$
\mathbf{f}(\mathbf{x})=(1-\mu) \mathbf{q}_{\ell}+\mu \mathbf{q}_{\ell+1} .
$$

Thus f maps $\partial P$ to $\partial Q$ in a piecewise linear fashion, mapping vertices and edges of $\partial P$ to corresponding vertices and edges of $\partial Q$.

Figure 2 shows examples of Wachspress and mean value mappings.

\section{Conditions for injectivity}

We start by deriving conditions on barycentric coordinates for injectivity: both sufficient conditions and necessary conditions.

Using basic results of real analysis, see for example [12], it can be shown that a sufficient condition for the injectivity of $\mathbf{f}$ in (10) is that its Jacobian $J(\mathbf{f})$ is strictly positive in $\bar{P}$. This motivates looking for an expression for $J(\mathbf{f})$ in terms of signed areas of triangles formed by vertices of $Q$. If $\mathbf{f}(\mathbf{x})=(f(\mathbf{x}), g(\mathbf{x}))$, and $\partial_{r} h(\mathbf{x}):=\partial h / \partial x^{r}, r=1,2$, the Jacobian of $\mathbf{f}$ is the determinant

$$
J(\mathbf{f})=\left|\begin{array}{cc}
\partial_{1} f & \partial_{1} g \\
\partial_{2} f & \partial_{2} g
\end{array}\right|
$$

Lemma 1 For any set of differentiable functions $\lambda_{1}, \ldots, \lambda_{n}$ satisfying (1),

$$
J(\mathbf{f})(\mathbf{x})=2 \sum_{1 \leq i<j<k \leq n} \operatorname{det}\left(\lambda_{i}, \lambda_{j}, \lambda_{k}\right)(\mathbf{x}) A\left(\mathbf{q}_{i}, \mathbf{q}_{j}, \mathbf{q}_{k}\right),
$$

where

$$
\operatorname{det}(a, b, c)=\left|\begin{array}{ccc}
a & b & c \\
\partial_{1} a & \partial_{1} b & \partial_{1} c \\
\partial_{2} a & \partial_{2} b & \partial_{2} c
\end{array}\right|
$$

Proof. Differentiating (1) and (10) gives the matrix identity

$$
\left(\begin{array}{ccc}
1 & f & g \\
0 & \partial_{1} f & \partial_{1} g \\
0 & \partial_{2} f & \partial_{2} g
\end{array}\right)=\left(\begin{array}{ccc}
\lambda_{1} & \cdots & \lambda_{n} \\
\partial_{1} \lambda_{1} & \cdots & \partial_{1} \lambda_{n} \\
\partial_{2} \lambda_{1} & \cdots & \partial_{2} \lambda_{n}
\end{array}\right)\left(\begin{array}{ccc}
1 & q_{1}^{1} & q_{1}^{2} \\
\vdots & \vdots & \vdots \\
1 & q_{n}^{1} & q_{n}^{2}
\end{array}\right)
$$

Applying the Cauchy-Binet theorem (see [1], formula (1.23)) to this equation and using the fact that the determinant of the matrix on the left equals $J(\mathbf{f})$, the result follows. 

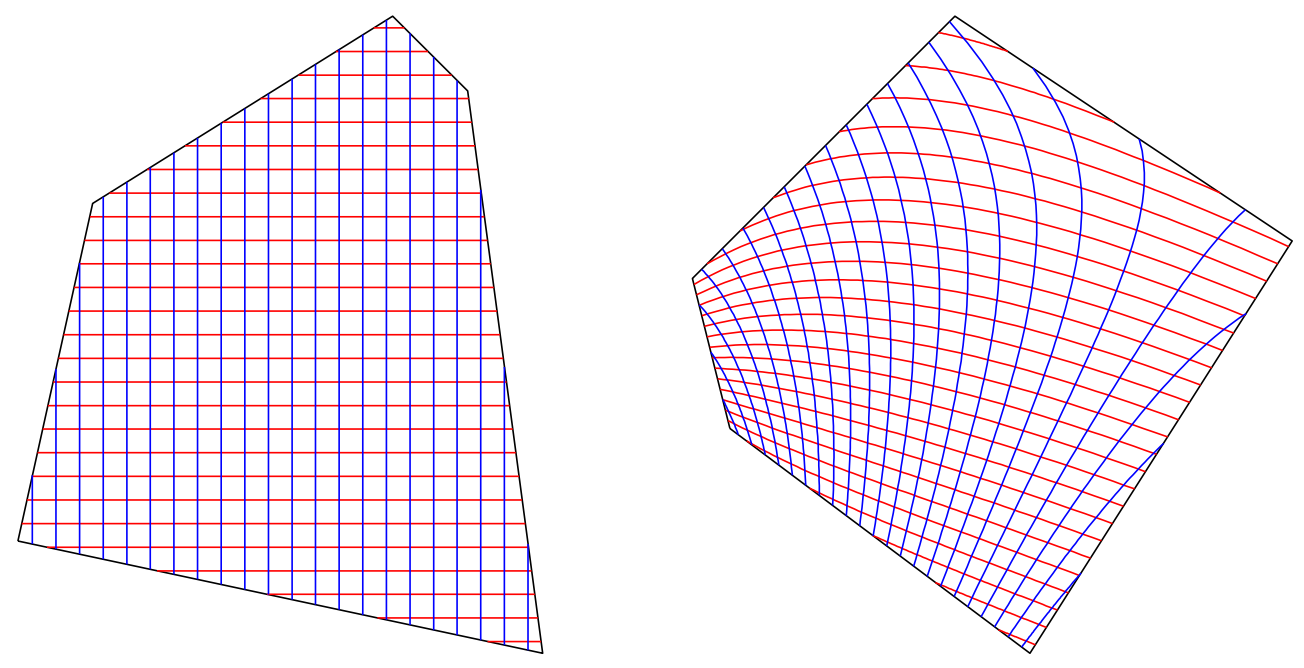

a)
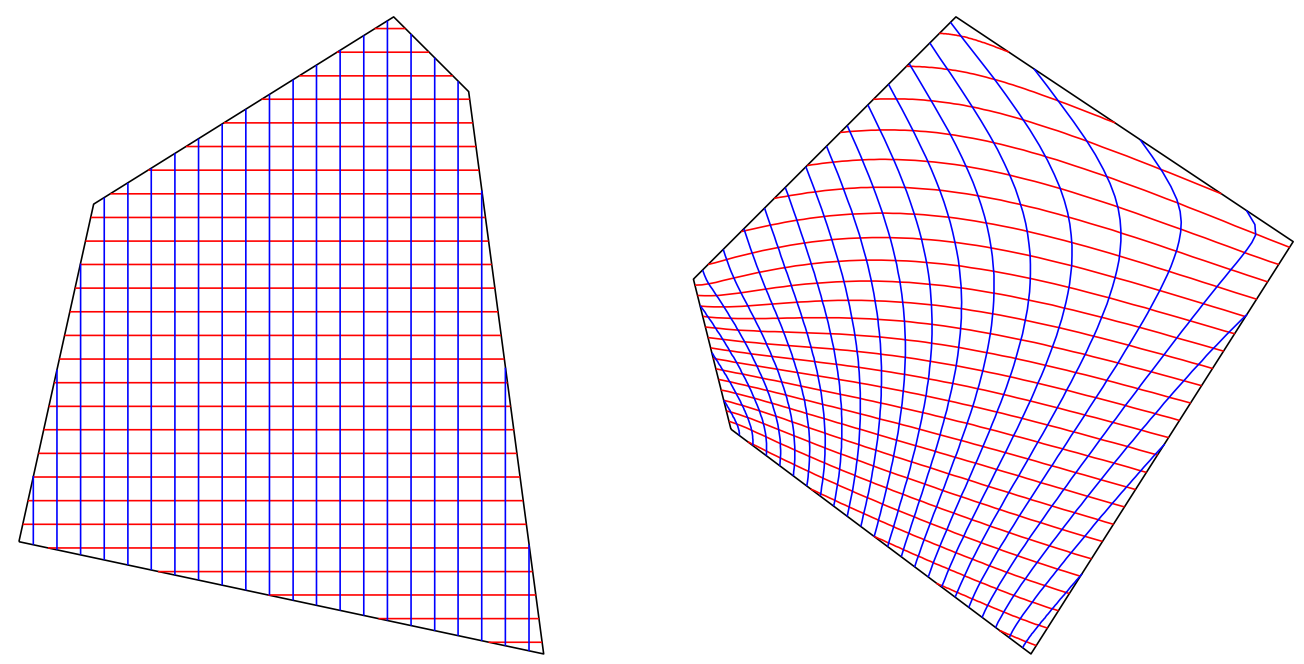

b)

Figure 2: Barycentric mappings between pentagons: a) Wachspress mapping, b) mean value mapping. 
Since $Q$ is strictly convex we have that $A\left(\mathbf{q}_{i}, \mathbf{q}_{j}, \mathbf{q}_{k}\right)>0$ whenever $1 \leq$ $i<j<k \leq n$ because the triangle $\left[\mathbf{q}_{i}, \mathbf{q}_{j}, \mathbf{q}_{k}\right]$ is positively oriented. Thus Lemma 1 leads to a sufficient condition for injectivity.

Theorem 1 If $\lambda_{1}, \ldots, \lambda_{n}$ are differentiable barycentric coordinates such that for all $\mathbf{x} \in \bar{P}$, $\operatorname{det}\left(\lambda_{i}, \lambda_{j}, \lambda_{k}\right)(\mathbf{x}) \geq 0$ for all $i, j, k$ with $1 \leq i<j<k \leq n$ and $\operatorname{det}\left(\lambda_{i}, \lambda_{j}, \lambda_{k}\right)(\mathbf{x})>0$ for some $i, j, k$ with $1 \leq i<j<k \leq n$ then $\mathbf{f}$ is injective.

Conversely, we derive necessary conditions for injectivity.

Theorem 2 If $\lambda_{1}, \ldots, \lambda_{n}$ are differentiable barycentric coordinates and $\mathbf{f}$ is injective then for all $r, s, t$ with $1 \leq r<s<t \leq n$,

$$
\sum_{r \leq i<s \leq j<t \leq k<n+r} \operatorname{det}\left(\lambda_{i}, \lambda_{j}, \lambda_{k}\right) \geq 0 \quad \text { in } P .
$$

Proof. If $\mathbf{f}$ is injective then $J(\mathbf{f}) \geq 0$ in $P$. Let $Q$ be the polygon with vertices

$$
\begin{gathered}
\mathbf{q}_{1}=\cdots=\mathbf{q}_{r-1}=(0,0), \quad \mathbf{q}_{r}=\cdots=\mathbf{q}_{s-1}=(1,0), \\
\mathbf{q}_{s}=\cdots=\mathbf{q}_{t-1}=(0,1), \quad \mathbf{q}_{t}=\cdots=\mathbf{q}_{n}=(0,0) .
\end{gathered}
$$

Then $A\left(\mathbf{q}_{i}, \mathbf{q}_{j}, \mathbf{q}_{k}\right)=1 / 2$ if $1 \leq i<r \leq j<s \leq k<t$ or $r \leq i<s \leq j<$ $t \leq k \leq n$ and zero otherwise. Substituting these values into (11) gives

$$
\sum_{1 \leq i<r \leq j<s \leq k<t} \operatorname{det}\left(\lambda_{i}, \lambda_{j}, \lambda_{k}\right)+\sum_{r \leq i<s \leq j<t \leq k \leq n} \operatorname{det}\left(\lambda_{i}, \lambda_{j}, \lambda_{k}\right) \geq 0 .
$$

Replacing $i, j, k$ by $k, i, j$ in the first sum and using the fact that

$$
\operatorname{det}\left(\lambda_{k}, \lambda_{i}, \lambda_{j}\right)=\operatorname{det}\left(\lambda_{i}, \lambda_{j}, \lambda_{k}\right),
$$

the first sum can be written as

$$
\sum_{1 \leq k<r \leq i<s \leq j<t} \operatorname{det}\left(\lambda_{i}, \lambda_{j}, \lambda_{k}\right)=\sum_{\substack{r \leq i<s \leq j<t \\ n+1 \leq k<n+r}} \operatorname{det}\left(\lambda_{i}, \lambda_{j}, \lambda_{k-n}\right) .
$$

Replacing $\lambda_{k-n}$ by $\lambda_{k}$, combining this second expression with the second sum in (14) gives (13). 
We have thus derived sets of sufficient conditions and necessary conditions for injectivity, based on the signs of the determinants $\operatorname{det}\left(\lambda_{i}, \lambda_{j}, \lambda_{k}\right)$. Finding conditions that are both sufficient and necessary could be difficult. Interestingly, a similar difficulty arose in $[3,4,6]$ when trying to characterize bases of functions that guarantee convexity preservation of curves: when the control polygon is convex, the curve should be too. So far, a characterization is not known. However, as we will see in the next section, the sufficient conditions of Theorem 1 are good enough to show that Wachspress mappings are injective and we use the necessary ones to help to derive a counterexample for mean value mappings.

For barycentric coordinates expressed in the form (4), the following identity helps to simplify the task of checking the signs of these determinants.

Lemma 2 For differentiable functions $a, b, c$, and $\mu$,

$$
\operatorname{det}(\mu a, \mu b, \mu c)=\mu^{3} \operatorname{det}(a, b, c) .
$$

Proof. Recalling (12), expand the second and third rows of the determinant $\operatorname{det}(\mu a, \mu b, \mu c)$ using the product rule. Then, subtracting $\partial_{1} \mu$ times the first row from the second row and subtracting $\partial_{2} \mu$ times the first row from the third row, gives the result.

\section{Wachspress mappings}

We now use the sufficient conditions of Theorem 1 to show that Wachspress mappings are injective.

Theorem 3 If $\lambda_{1}, \ldots, \lambda_{n}$ are the Wachspress coordinates $\left(4^{-5}\right)$ then $\mathbf{f}$ is injective.

Proof. The proof uses Theorem 1 and consists of two steps dealing with $\mathbf{x} \in P$ and $\mathbf{x} \in \partial P$, respectively.

Step 1: First let $\mathbf{x} \in P$ and choose $i, j, k$ such that $1 \leq i<j<k \leq n$. We will show that $\operatorname{det}\left(\lambda_{i}, \lambda_{j}, \lambda_{k}\right)(\mathbf{x})>0$. Since $\mathbf{x}$ is not on the boundary of $P$ we can define $\lambda_{i}$ in (4) using the rational weight function $w_{i}$ of (6), i.e., as $\lambda_{i}=\mu w_{i}$, where $\mu=1 / \sum_{j} w_{j}>0$, and then by Lemma 2 , it is sufficient to show that $\operatorname{det}\left(w_{i}, w_{j}, w_{k}\right)(\mathbf{x})>0$. To this end, observe that

$$
\operatorname{det}\left(w_{i}, w_{j}, w_{k}\right)=w_{i} \nabla w_{j} \times \nabla w_{k}+w_{j} \nabla w_{k} \times \nabla w_{i}+w_{k} \nabla w_{i} \times \nabla w_{j},
$$


where $\nabla w:=\left(\partial_{1} w, \partial_{2} w\right)$ and $\mathbf{p} \times \mathbf{q}:=p^{1} q^{2}-p^{2} q^{1}$. Using (3), differentiation of $A_{i}(\mathbf{x})$ with respect to $x^{1}$ and $x^{2}$ gives

$$
\nabla A_{i}=\frac{1}{2} \operatorname{rot}\left(\mathbf{p}_{i+1}-\mathbf{p}_{i}\right)
$$

where $\operatorname{rot}\left(p^{1}, p^{2}\right):=\left(-p^{2}, p^{1}\right)$, a rotation through a positive angle of $\pi / 2$. Therefore, from (6), we have

$$
\nabla w_{i}=-w_{i}\left(\frac{\operatorname{rot}\left(\mathbf{p}_{i}-\mathbf{p}_{i-1}\right)}{2 A_{i-1}(\mathbf{x})}+\frac{\operatorname{rot}\left(\mathbf{p}_{i+1}-\mathbf{p}_{i}\right)}{2 A_{i}(\mathbf{x})}\right)
$$

and it follows that

$$
\nabla w_{i} \times \nabla w_{j}=w_{i} w_{j} S_{i j}
$$

where

$$
S_{i j}=R_{i-1, j-1}+R_{i-1, j}+R_{i, j-1}+R_{i j}
$$

and

$$
R_{i j}=\frac{\operatorname{rot}\left(\mathbf{p}_{i+1}-\mathbf{p}_{i}\right) \times \operatorname{rot}\left(\mathbf{p}_{j+1}-\mathbf{p}_{j}\right)}{4 A_{i}(\mathbf{x}) A_{j}(\mathbf{x})}=\frac{\left(\mathbf{p}_{i+1}-\mathbf{p}_{i}\right) \times\left(\mathbf{p}_{j+1}-\mathbf{p}_{j}\right)}{4 A_{i}(\mathbf{x}) A_{j}(\mathbf{x})} .
$$

Substituting (18) into (16) gives

$$
\operatorname{det}\left(w_{i}, w_{j}, w_{k}\right)=w_{i} w_{j} w_{k}\left(S_{i j}+S_{j k}+S_{k i}\right),
$$

where we have used the fact that $R_{i j}=-R_{j i}$ and consequently $S_{i j}=-S_{j i}$. Now, each of the three $S$ terms expands into four $R$ terms, giving twelve $R$ terms altogether. We can group these twelve terms into four groups of three such that each group has the form

$$
T_{p q r}:=R_{p q}+R_{q r}+R_{r p} .
$$

There are two ways of doing this. One is

$$
\operatorname{det}\left(w_{i}, w_{j}, w_{k}\right)=w_{i} w_{j} w_{k}\left(T_{i, j, k}+T_{i, j-1, k-1}+T_{i-1, j, k-1}+T_{i-1, j-1, k}\right),
$$

and the other is

$$
\operatorname{det}\left(w_{i}, w_{j}, w_{k}\right)=w_{i} w_{j} w_{k}\left(T_{i-1, j-1, k-1}+T_{i, j, k-1}+T_{i, j-1, k}+T_{i-1, j, k}\right) .
$$




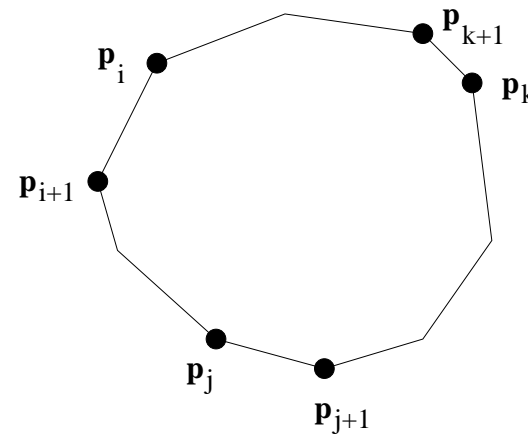

(a)

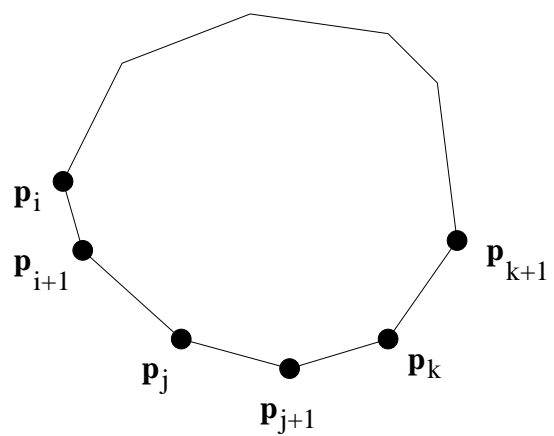

(b)

Figure 3: At least two cross products are positive

Now, since $R_{i i}=0$, we see that $T_{p q r}=0$ whenever $p=q$ or $q=r$. Therefore, using either expression, we see that $\operatorname{det}\left(w_{i}, w_{j}, w_{k}\right)>0$ if

$$
T_{i j k}>0, \quad 1 \leq i<j<k \leq n .
$$

To show this, observe from the cross product in (19) and from the convexity of $P$ that at least two of the terms $R_{i j}, R_{j k}, R_{k i}$ must be strictly positive. We may assume without loss of generality that these are $R_{i j}$ and $R_{j k}$. If the third term $R_{k i}$ is non-negative, as illustrated in Figure $3(\mathrm{a})$, then $T_{i j k}>0$ as claimed. Thus it remains to treat the case that $R_{k i}<0$, as illustrated in Figure 3(b). To this end, with $L_{\alpha}$ denoting the infinite line that extends the line segment $\left[\mathbf{p}_{\alpha}, \mathbf{p}_{\alpha+1}\right]$, consider the three lines $L_{i}, L_{j}, L_{k}$. They intersect in three points: $\mathbf{r}_{1}$ the intersection of $L_{i}$ and $L_{j} ; \mathbf{r}_{2}$ the intersection of $L_{i}$ and $L_{k}$; and $\mathbf{r}_{3}$ the intersection of $L_{j}$ and $L_{k}$; see Figure 4 . Then, recalling the 'half the base times the height' rule for the area of a triangle, it is easy to see that

$$
\begin{aligned}
\frac{\mathbf{p}_{i+1}-\mathbf{p}_{i}}{A_{i}(\mathbf{x})} & =\frac{\mathbf{r}_{2}-\mathbf{r}_{1}}{A\left(\mathbf{x}, \mathbf{r}_{1}, \mathbf{r}_{2}\right)}, \\
\frac{\mathbf{p}_{j+1}-\mathbf{p}_{j}}{A_{j}(\mathbf{x})} & =\frac{\mathbf{r}_{3}-\mathbf{r}_{1}}{A\left(\mathbf{x}, \mathbf{r}_{1}, \mathbf{r}_{3}\right)}, \\
\frac{\mathbf{p}_{k+1}-\mathbf{p}_{k}}{A_{k}(\mathbf{x})} & =\frac{\mathbf{r}_{3}-\mathbf{r}_{2}}{A\left(\mathbf{x}, \mathbf{r}_{2}, \mathbf{r}_{3}\right)},
\end{aligned}
$$

and it follows from (19) that

$$
R_{i j}=\frac{A\left(\mathbf{r}_{1}, \mathbf{r}_{2}, \mathbf{r}_{3}\right)}{2 A\left(\mathbf{x}, \mathbf{r}_{1}, \mathbf{r}_{2}\right) A\left(\mathbf{x}, \mathbf{r}_{1}, \mathbf{r}_{3}\right)}
$$




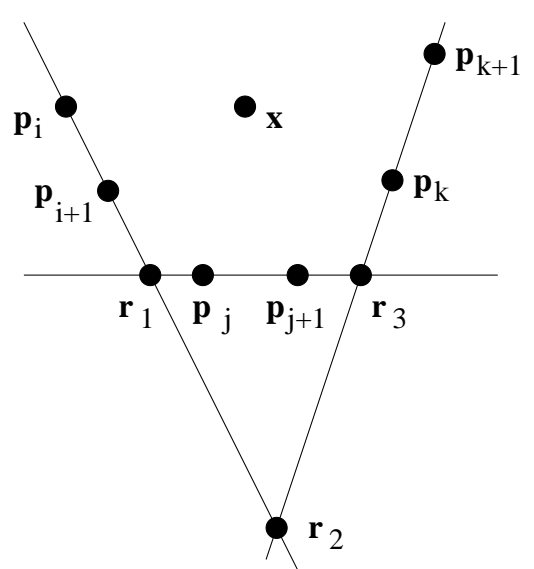

Figure 4: Intersections of three lines

$$
\begin{aligned}
R_{j k} & =\frac{A\left(\mathbf{r}_{1}, \mathbf{r}_{2}, \mathbf{r}_{3}\right)}{2 A\left(\mathbf{x}, \mathbf{r}_{1}, \mathbf{r}_{3}\right) A\left(\mathbf{x}, \mathbf{r}_{2}, \mathbf{r}_{3}\right)} \\
R_{k i} & =\frac{-A\left(\mathbf{r}_{1}, \mathbf{r}_{2}, \mathbf{r}_{3}\right)}{2 A\left(\mathbf{x}, \mathbf{r}_{2}, \mathbf{r}_{3}\right) A\left(\mathbf{x}, \mathbf{r}_{1}, \mathbf{r}_{2}\right)}
\end{aligned}
$$

So

$$
T_{i j k}=\frac{\left(A\left(\mathbf{r}_{1}, \mathbf{r}_{2}, \mathbf{r}_{3}\right)\right)^{2}}{2 A\left(\mathbf{x}, \mathbf{r}_{1}, \mathbf{r}_{2}\right) A\left(\mathbf{x}, \mathbf{r}_{1}, \mathbf{r}_{3}\right) A\left(\mathbf{x}, \mathbf{r}_{2}, \mathbf{r}_{3}\right)}>0 .
$$

This shows that $\operatorname{det}\left(w_{i}, w_{j}, w_{k}\right)(\mathbf{x})>0$ for $\mathbf{x} \in P$.

Step 2: It remains to deal with $\mathbf{x} \in \partial P$, which we may assume to be as in (8) for some $\ell$ and $\mu \in[0,1)$. Since the $\lambda_{i}$ are continuous on the whole of $\bar{P}$ and $\operatorname{det}\left(\lambda_{i}, \lambda_{j}, \lambda_{k}\right)(\mathbf{x})>0$ on $P$, we know that $\operatorname{det}\left(\lambda_{i}, \lambda_{j}, \lambda_{k}\right)(\mathbf{x}) \geq 0$ by Step 1 . However, for general $n$, it is possible that there are some triples $i, j, k$ with $1 \leq i<j<k \leq n$ such that $\operatorname{det}\left(\lambda_{i}, \lambda_{j}, \lambda_{k}\right)(\mathbf{x})=0$. In fact, recalling (12), this is the case when none of $i, j, k$ equal either $\ell$ or $\ell+1$, for then $\lambda_{i}(\mathbf{x})=$ $\lambda_{j}(\mathbf{x})=\lambda_{k}(\mathbf{x})=0$. We will, however, show that $\operatorname{det}\left(\lambda_{\ell-1}, \lambda_{\ell}, \lambda_{\ell+1}\right)(\mathbf{x})>0$ which is sufficient for injectivity by Theorem 1 . To simplify this task we again appeal to Lemma 2 but this time must use the polynomial $w_{i}$. We will show that $\operatorname{det}\left(w_{\ell-1}, w_{\ell}, w_{\ell+1}\right)(\mathbf{x})>0$ with $w_{i}$ as in (5). From (16), we can write the determinant as

$$
\operatorname{det}\left(w_{\ell-1}, w_{\ell}, w_{\ell+1}\right)=w_{\ell-1} \nabla w_{\ell} \times \nabla w_{\ell+1}+w_{\ell}^{2} \nabla\left(\frac{w_{\ell+1}}{w_{\ell}}\right) \times \nabla w_{\ell-1},
$$


valid as long as $w_{\ell} \neq 0$. This expression is convenient because

$$
\frac{w_{\ell+1}}{w_{\ell}}=\frac{C_{\ell+1}}{C_{\ell}} \frac{A_{\ell-1}}{A_{\ell+1}}
$$

and, using (17),

$$
\nabla\left(\frac{A_{\ell-1}}{A_{\ell+1}}\right)=\frac{\operatorname{rot}\left(\mathbf{p}_{\ell}-\mathbf{p}_{\ell-1}\right)}{2 A_{\ell+1}}-\frac{A_{\ell-1} \operatorname{rot}\left(\mathbf{p}_{\ell+1}-\mathbf{p}_{\ell}\right)}{2 A_{\ell+1}^{2}} .
$$

Using these observations, we now evaluate (20) at $\mathbf{x}=(1-\mu) \mathbf{p}_{\ell}+\mu \mathbf{p}_{\ell+1}$. Since $w_{\ell-1}(\mathbf{x})=0$ and

$$
\nabla w_{\ell-1}(\mathbf{x})=K \operatorname{rot}\left(\mathbf{p}_{\ell+1}-\mathbf{p}_{\ell}\right)
$$

where

$$
K=C_{\ell-1} \prod_{j \neq \ell-1, \ell, \ell+1} A_{j}(\mathbf{x})>0
$$

we find

$$
\operatorname{det}\left(w_{\ell-1}, w_{\ell}, w_{\ell+1}\right)(\mathbf{x})=K_{1} A\left(\mathbf{p}_{\ell-1}, \mathbf{p}_{\ell}, \mathbf{p}_{\ell+1}\right)+K_{2} A\left(\mathbf{p}_{\ell}, \mathbf{p}_{\ell+1}, \mathbf{p}_{\ell+2}\right),
$$

where

$$
K_{1}=K \frac{C_{\ell+1}}{C_{\ell}} \frac{w_{\ell}^{2}(\mathbf{x})}{A_{\ell+1}(\mathbf{x})}>0
$$

and

$$
K_{2}=K \frac{C_{\ell+1}}{C_{\ell}} \frac{w_{\ell}^{2}(\mathbf{x}) A_{\ell-1}(\mathbf{x})}{A_{\ell+1}^{2}(\mathbf{x})}>0 .
$$

\section{Mean value mappings}

Mean value mappings are defined by (10), (4) and (7). Following numerous numerical tests, we conjecture that they are always injective when $n=4$. However, we have discovered counterexamples when $n=5$. These were found by exploiting Theorem 2 . 

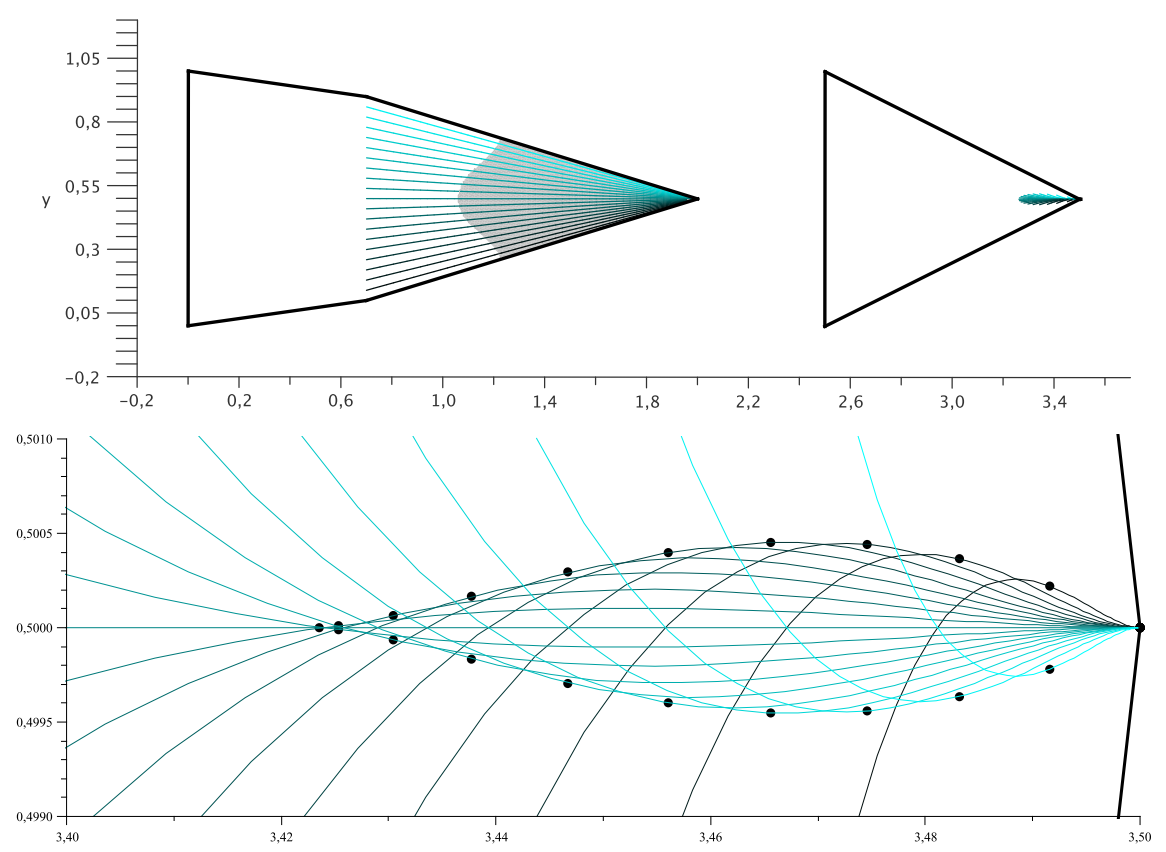

Figure 5: Counterexample 1: Mean value mapping between two convex polygons. Top: The gray region $G$ corresponds to negative Jacobian in $P$. Bottom: The image of $G$, the foldover area, is bounded by the black dots in $Q$. Only a scaled part of $Q$ near its vertex $\mathbf{q}_{3}$ is shown.
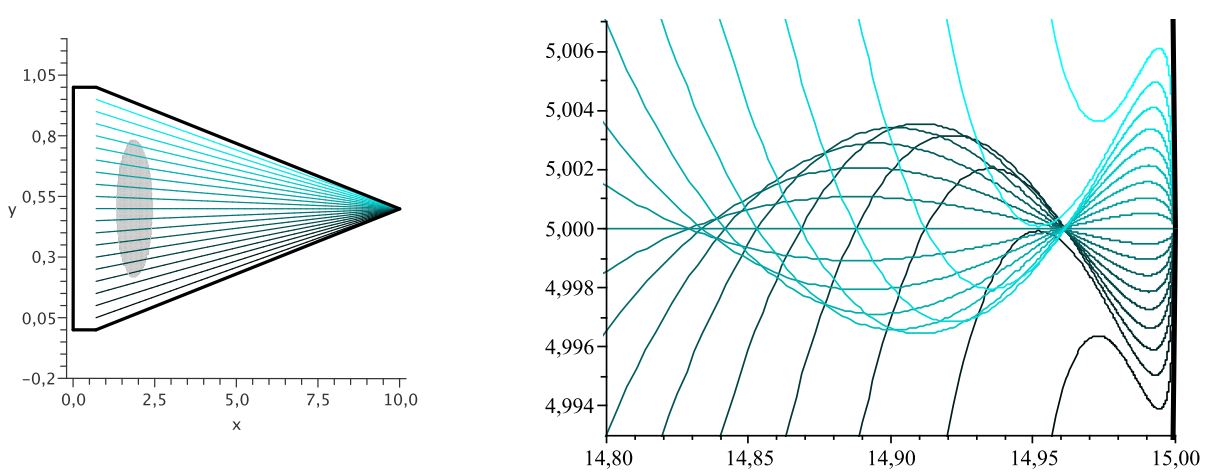

Figure 6: Counterexample 2: A foldover of a mean value mapping over two strictly convex polygons. $P$ is non-uniformly scaled and only a part of $Q$ is shown in order to emphasize the foldover. 


\subsection{Counterexample 1}

Let us suppose that $P$ is given by $\mathbf{p}_{1}=(0,0), \mathbf{p}_{2}=(0.7,0.1), \mathbf{p}_{3}=(2,0.5)$, $\mathbf{p}_{4}=(0.7,0.9)$ and $\mathbf{p}_{5}=(0,1)$. Moreover, let $\mathbf{x}=(1.2,0.5) \in P$. By evaluating $\operatorname{det}\left(\lambda_{i}, \lambda_{j}, \lambda_{k}\right)(\mathbf{x})$ for the mean value coordinates of $P$ one obtains that

$$
\begin{aligned}
& \operatorname{det}\left(\lambda_{1}, \lambda_{2}, \lambda_{5}\right)(\mathbf{x})=-0.00011838 \\
& \operatorname{det}\left(\lambda_{1}, \lambda_{3}, \lambda_{5}\right)(\mathbf{x})=-0.00040841 \\
& \operatorname{det}\left(\lambda_{1}, \lambda_{4}, \lambda_{5}\right)(\mathbf{x})=-0.00011838
\end{aligned}
$$

Therefore,

$$
\operatorname{det}\left(\lambda_{1}, \lambda_{2}, \lambda_{5}\right)(\mathbf{x})+\operatorname{det}\left(\lambda_{1}, \lambda_{3}, \lambda_{5}\right)(\mathbf{x})+\operatorname{det}\left(\lambda_{1}, \lambda_{4}, \lambda_{5}\right)(\mathbf{x})=-0.00064517 .
$$

As this value is negative, it violates the necessary conditions of Theorem 2 for $n=5, r=1, s=2$ and $t=5$. Consequently, by choosing $Q$ to be, for example, given by $\mathbf{q}_{1}=(2.5,0), \mathbf{q}_{2}=\mathbf{q}_{3}=\mathbf{q}_{4}=(3.5,0.5)$ and $\mathbf{q}_{5}=(2.5,1)$, we arrive at an example of a mean value mapping over convex polygons where the Jacobian is negative at some interior points. Indeed, $J(\mathbf{f})(\mathbf{x})=-0.00064517$.

Figure 5 depicts the situation and shows the region of $P$ where the corresponding Jacobian is negative. Moreover, it shows a star of line segments in $P$. One can see the intersections of their images in Figure $5-$ a fact that contradicts the injectivity of mean value mappings over convex polygons.

\subsection{Counterexample 2}

In this second example we assume that $P$ has vertices $\mathbf{p}_{1}=(0,0), \mathbf{p}_{2}=$ $(0.7,0), \mathbf{p}_{3}=(10,0.5), \mathbf{p}_{4}=(0.7,0.1), \mathbf{p}_{5}=(0,1)$ and $Q$ is given by $\mathbf{q}_{1}=$ $(13,0), \mathbf{q}_{2}=(14.999,4.99), \mathbf{q}_{3}=(15,5), \mathbf{q}_{4}=(14.999,5.01)$ and $\mathbf{q}_{5}=$ $(13,10)$.

Note that in this example both $P$ and $Q$ are strictly convex polygons. Still, Figure 6 shows that the corresponding mean value mapping is not injective.

\section{References}

[1] T. Ando, Totally positive matrices, Linear Algebra Appl. 90 (1987), $165-219$. 
[2] A. Belyaev, On transfinite barycentric coordinates, in Eurographics symposium on geometry processing, K. Polthier and A. Sheffer (eds.), 2006, pp 89-99.

[3] J. M. Carnicer, M. Garcia-Esnaola, and J. M. Peña, Convexity of rational curves and total positivity, J. Comp. Appl. Math. 71 (1996), $365-382$.

[4] J. M. Carnicer and M. Garcia-Esnaola, Global convexity of curves and polygons, Comp. Aided Geom. Design 15 (1998), 893-907.

[5] C. Dyken and M. S. Floater, Transfinite mean value interpolation, to appear in Comp. Aided Geom. Design.

[6] M. S. Floater, Total positivity and convexity preservation, J. Approx. Theory 96 (1999), 46-66.

[7] M. S. Floater, Mean value coordinates, Comp. Aided Geom. Design 20 (2003), 19-27.

[8] M. S. Floater, K. Hormann, and G. Kós, A general construction of barycentric coordinates over convex polygons, Adv. in Comp. Math. 24 (2006), 311-331.

[9] M. S. Floater, G. Kos, and M. Reimers, Mean value coordinates in 3D, Comp. Aided Geom. Design 22 (2005), 623-631.

[10] K. Hormann and M. S. Floater, Mean value coordinates for arbitrary planar polygons, ACM Trans. on Graphics 25 (2006), 1424-1441.

[11] W. J. Gordon and J. A. Wixom, Pseudo-harmonic interpolation on convex domains, SIAM J. Numer. Anal. 11 (1974), 909-933.

[12] H. Kestelman, Mappings with non-vanishing Jacobian, Amer. Math. Monthly 78 (1971), 662-663.

[13] M. Meyer, A. Barr, H. Lee, and M. Desbrun, Generalized barycentric coordinates on irregular polygons, Journal of graphics tools 7 (2002), $13-22$.

[14] T. Ju, S. Schaefer, and J. Warren, Mean value coordinates for closed triangular meshes, ACM Trans. on Graphics 24 (2005), 561-566. 
[15] T. Ju, S. Schaefer, J. Warren, and M. Desbrun, A geometric construction of coordinates for convex polyhedra using polar duals, in Geometry Processing 2005, M. Desbrun and H. Pottman (eds.), Eurographics Association 2005, 181-186.

[16] T. Langer, A. Belyaev, and H.-P. Seidel, Spherical barycentric coordinates, in Eurographics symposium on geometry processing, K. Polthier and A. Sheffer (eds.), 2006, pp 81-88.

[17] S. L. Lee, Mean value representations and curvatures of compact convex hypersurfaces, preprint, 2007.

[18] Y. Lipman, J. Kopf, D. Cohen-Or, and D. Levin, GPU-assisted positive mean value coordinates for mesh deformation, in Symposium on Geometry Processing, 2007.

[19] S. Schaefer, T. Ju, and J. Warren, A unified integral construction for coordinates over closed curves, Comp. Aided Geom. Design 24 (2007), 481-493.

[20] E. L. Wachspress, A rational finite element basis, Academic Press, New York, 1975.

[21] J. Warren, Barycentric coordinates for convex polytopes, Adv. in Comp. Math. 6 (1996), 97-108.

[22] J. Warren, S. Schaefer, A. Hirani, and M. Desbrun, Barycentric coordinates for convex sets, Adv. in Comp. Math. 27 (2007), 319-338. 